\title{
Clients' subjective experience of their participation in rehabilitation at an out-patient community rehabilitation center
}

\author{
Maatje Kloppers, Qualifications: BOT (SU), MOT (SU) \\ Senior Occupational Therapist, Bishop Lavis Rehab Centre, Occupational Therapy Division, Faculty of Medicine and Health \\ Sciences, Stellenbosch University
}

\author{
Blanche Pretorius, Nat Dip OT (Vona du Toit College), B.A. Hons in Communications (UNISA), Post- \\ graduate Dip in Tertiary Education (UP), M Ed in Higher Education (SU), Post-graduate Dip in Programme \\ Monitoring and Evaluation (SU) \\ Part-time lecturer, Occupational Therapy Division, Stellenbosch University
}

Elizabeth Daphné Vlok, BOT (SU), MOT (SU)

Lecturer, Occupational Therapy Division, Stellenbosch University

Introduction: Rehabilitation services in South Africa are governed by various policies. It is important to evaluate these services to assess if the services are achieving their aims as set out in these policies. The evaluation should include the outcomes that clients achieve by participating in rehabilitation services.

Method: The findings presented were obtained as part of a larger mixed methods descriptive study and this paper will focus on clients' subjective experience of participating in rehabilitation. Convenience sampling was used to select 78 clients from the five most prevalent diagnostic groups seen at the center. Semi-structured interviews were conducted, transcribed and analysed by means of basic content analysis.

Results: Clients commented on the emotional impact that participating in rehabilitation had had on them and on the physical changes it had brought about. Clients experienced a high level of satisfaction with the service and enjoyed participating in rehabilitation. The knowledge, skills and confidence that they gained enabled them to be more independent in Daily life activities and to share this knowledge with others in their community.

Conclusions: The results emphasise the importance of providing rehabilitation services for persons with disabilities that are based in their community. It will be useful in further service and policy planning for persons with disabilities.

Keywords: rehabilitation in community, outcomes, subjective experience

\section{INTRODUCTION}

In this article motivation regarding the evaluation of the outcomes of rehabilitation services focusing on the importance of subjective data from clients with disabilities, will be described. Data obtained as part of the study for a Master's degree will be presented in order to illustrate that clients benefited psychologically as well as physically from participation in the rehabilitation services offered at a community rehabilitation centre in the Cape Town area.

Rehabilitation services in South Africa and the Western-Cape are governed by policies such as the National Rehabilitation Policy(NRP)', the White paper on an Integrated National Disability Policy(WPINDP) ${ }^{2}$; the Western Capes Healthcare 2010 plan $^{3}$ through the Comprehensive Service Plan(CSP) ${ }^{4}$ as well as the Norms and Standards for Primary Healthcare ${ }^{5}$. The Department of Health is currently busy planning and implementing the Re-engineering of Primary Healthcare as part of the Healthcare 2020 policy $^{6}$.

It is important to evaluate rehabilitation services to understand the impact of these policies and to assess if the services being offered are achieving the aims as set out in them. When evaluating rehabilitation services information should be gathered on the outcomes that clients achieve through their participation in rehabilitation services. Kayes and McPherson ${ }^{7}$ argued for the importance of supplementing objective information on clients' functioning with subjective information as objective measuring instruments do not always adequately reflect abilities and cannot capture a person's feelings regarding his or her dependence (or independence). This research set out to obtain information on the user outcomes of the rehabilitation service users.

\section{Contextual background}

The community that participated in the research is situated in the densely populated Cape Flats area in the Western Cape - a historically under-privileged, low socio-economic area. The area was previously classified under the Group Areas Act as a community for persons classified as non-white. Approximately a third of the population is unemployed ${ }^{8-10}$.

In the Western Cape the rate of unemployed persons with disabilities is $3 \%$ higher than for non-disabled persons ${ }^{10}$. The 2011 Census determined that $4.6 \%$ of Western Cape Province residents have a disability compared to $5.1 \%$ nationally ${ }^{8}$. Physical disabilities are the second most prevalent type of disability $(29,6 \%$ of all disability types) ${ }^{9}$. Persons with disabilities thus comprise a large percentage of the total population. Detailed information regarding their functional performance is needed to effectively plan for services to meet their needs ${ }^{10}$.

The local rehabilitation center strives to meet these needs by rendering a free comprehensive primary level out-patient rehabilitation service and prides itself on the strength of its volunteers who play a significant role in the service delivered to community members. Five volunteers spend an average of six hours per week to enrich service delivery by providing much needed assistance to the 
groups at the rehabilitation center and in the community, preparation for craft groups and assisting with fundraising for the centre.

The struggles faced at the center are nothing new to healthcare professionals working within a resource-constrained environment. These constraints include staff and space shortages, limited equipment and materials as well as poor client compliance. Despite these challenges the rehabilitation center delivers an interdisciplinary service comprising the four professions of Occupational Therapy, Physiotherapy, Dietetics and Speech, Language and Hearing therapy. The rehabilitation center also functions as an undergraduate training facility for students in the above-mentioned professions.

This study was done with the aim of evaluating the outcomes that clients achieve through their participation in the rehabilitation service. The subjective data gathered on clients' opinions on the difference therapy made in their lives will be the focus of this article.

\section{LITERATURE REVIEW}

The South African healthcare system has been undergoing transformation since the first democratic elections in 1994. In the Western Cape Province this change was initially guided by the Healthcare 2010 plan $^{3}$ and more specifically the CSP. The Re-engineering of Primary Healthcare and Healthcare 2020 policies have since been drafted $^{6}$. The goal of these directives has been to shift the focus of healthcare services from a tertiary level to a primary level; thus into the community, especially to previously disadvantaged communities where comprehensive healthcare services were not previously available.

Since this transformation the delivery of rehabilitation services has been guided by a variety of guidelines and policies. The minimum standards and norms to which rehabilitation services in the primary sector of South Africa must adhere are made explicit in a variety of white papers and policies ${ }^{1-6}$. From these sources the norms and standards can be summarised as follows:

* All clients discharged from hospital or referred for rehabilitation must undergo a screening or functional assessment.

* A low intensity rehabilitation service must be provided for at least an hour a day (not necessarily every day), by at least a physiotherapist and/or an Occupational therapist in the natural environment or a simulated environment that closely matches the environment in which the skill must be performed.

* Basic functional skills training must be available in activities of daily life especially in terms of self-care, mobility, safety and communication, so that a basic level of independence - with help where necessary - can be achieved.

* Relief of acute symptoms through therapy.

* Therapeutic and support groups must be available.

* Education, training and support to clients, their family and primary care giver must be provided.

* Appropriate assistive devices must be affordable and readily available to anyone that might benefit from the correcting affected areas and thus limiting the effect of the disability and enabling the user to reach an optimal level of functioning in all areas of Activities of Daily Life (ADL).

$*$ Information regarding the prevention of disability and health promotion must be provided to clients, and to their families and primary care givers to empower them to make informed decisions regarding their health.

* There must be a functioning referral system between resources in the community to connect clients and their families to resources in order to enable easier reintegration into their home and community.

These policy documents also indicate that monitoring and evaluation of healthcare services form an integral part of service delivery ${ }^{4}$, but to date there have not been any formal or informal evaluations or reporting on rehabilitation services, except for 'headcount statistics'.

The change in focus of healthcare service delivery towards a primary level prompted a similar shift in rehabilitation services. The rehabilitation service at the rehabilitation center is shaped by the Community Based Rehabilitation (CBR) model which integrates primary healthcare principles into the rehabilitation service. This includes treatment delivered in clients' homes and providing support and training to home-based carers that work in the community with persons with dissabililities.

McColl et al. ${ }^{11: 1525}$ describes the integration of rehabilitation services and a primary healthcare approach as a positive service geared ".... to delivering rehabilitation services in the developing world, where both material and human resources are scarce". This is achieved by the rehabilitation professionals who advocate for the interests of people with disabilities, help to identify community resources and secure support for them. This approach usually has a wide and far- reaching effect, especially with regards to the awareness of disability that leads to improved accessibility to and inclusion in communities'". In 2004 the updated version of the 1994 'Joint position paper on CBR: A strategy for rehabilitation, equalisation of opportunities, poverty reduction and social inclusion of people with disabilities'12 was issued by the World Health Organisation (WHO) to serve as a framework and give international guidance regarding the planning and implementation of CBR. The WHO emphasises the role that CBR can play in reducing the impact that disability has on a person's life, as well as - from a social model perspective - the positive change in the attitude of communities towards persons with disabilities. Rehabilitation should also result in persons with disabilities experiencing dignity as well as exercising their human rights ${ }^{13}$.

Velema, Ebenso \& Fuzikawa ${ }^{14}$ analysed 29 reports from 22 middle to low socio-economic countries (including South Africa) compiled in the period 1997 to 2007 , to determine what progress had been reported after implementation and evaluation of their rehabilitation-in-the-community-programmes. They reported the following:

* Improvement was noted mainly in clients' independence, mobility and communication.

* Clients reported that the rehabilitation-in-the-community programme positively changed the community's perception of disability, that they felt more included in the community and that the community's level of acceptance of the disabled had improved.

* There was an increase in spending of financial resources to provide for the needs of the disabled.

These areas of progress from a diverse spectrum of programmes are helpful norms against which other rehabilitation-in-the-community outcomes may be evaluated. Aspects of these diverse programmes overlap with services offered at the rehabilitation center under consideration - such as providing intervention in the home environment, training of volunteers and carers and general education to community members on prevention of illness and disability.

The problems highlighted by the literature on the evaluation of rehabilitation programmes are especially related to comparability of data due to the diverse nature of rehabilitation-in-the-community programmes $^{15}$ and that no uniform structure or terminology is used in evaluations ${ }^{14,16,17}$. This leads to a shortage in internationally comparable data regarding functioning and disability ${ }^{18}$.

McNamara ${ }^{19}$ identified three types of approaches to evaluation, namely goal, process and outcome-based evaluation, with an emphasis of evaluation of programme outcomes. Cameron ${ }^{20}$ warns against the use of process evaluation in rehabilitation as it does not accurately reflect effectiveness (for example, that the number of clients seen by a therapist does not reflect the effectiveness of the contact session with the clients). According to Young ${ }^{21}$, outcomebased evaluation focuses on the changes that take place in clients' lives, rather than changes in the programme itself - i.e. an increase in knowledge, skills and attitude or in improved standards of living ${ }^{19}$. Hoenig et al. ${ }^{16}$ argued that in addition to the previously mentioned definition it is also important to assess clients' satisfaction with the service and to evaluate their use of these services. 
In order to evaluate rehabilitation policies and to plan for effective and efficient service delivery, it is important to assess outcomes from the perspective of the users of a particular service. When assessing clients' functioning it should be noted that clients' subjective experiences of their functioning do not always match the objective measuring of their functioning ${ }^{22}$. It is therefore of the utmost importance to also gather subjective information when assessing a person's functioning ${ }^{7}$.

Finkenflügel, Cornielje \& Velema ${ }^{23}$ suggested that data obtained from disability studies be used in the planning of services and to guide policy changes and development. Because the implementation of rehabilitation services, as an intended part of a comprehensive Primary Health Care Approach, has not received sufficient emphasis, the outcomes of services such as the one offered by the rehabilitation center where this study was conducted is of particular importance.

\section{METHODOLOGY}

The findings presented in this article were obtained as part of a larger mixed methods descriptive study. The sequential study design comprised a quantitative component in which a pre-test post-test assessment was done using the World Health Organisation's International Classification of Functioning framework ${ }^{24}$. This was followed by a qualitative component. The focus of this paper will be on the latter.

\section{Aim}

The aim of the study reported on here was to determine the clients' subjective experience of the change in their daily lives after participating in the rehabilitation services.

\section{Data collection}

Data were obtained in the form of individual semi-structured interviews. Questions used in the interviews were refined in a pilot study and a fieldworker was trained to conduct them. The decision to use one of the rehabilitation center's volunteers (who come from the same community) for data collection was taken in an attempt to reduce the power imbalance during interviews and thereby create the opportunity for clients to freely share and express their true feelings. It was anticipated that the researcher's role as therapist and co-manager of the center would restrict participants' freedom to share their views, especially if these were negative.

The interviews comprised four open ended questions that were selected to elicit clients' personal perspectives on outcomes reached, as well as their subjective opinion of the rehabilitation experience. Clients were asked to comment on their functioning difficulties before and after rehabilitation and to describe in their own words what participation in rehabilitation meant to them. Interviews took on average 10 minutes to complete and were conducted either at the clients' home or at the rehabilitation center - depending on what would be convenient for the client. All interviews were recorded and transcribed in preparation for analysis.

\section{Study sample}

Convenience sampling was used to select 78 clients who were referred to the rehabilitation center during the period $01 / 09 / 2010$ to $3 \mathrm{I} / 03 / \mathrm{I}$ I from the five most prevalent diagnostic groups. The most prevalent diagnostic groups were identified from the center's statistics over a period of one year and were as follows: Back pain, CVA, Arthritis, Upper limb injuries and Lower limb injuries. All clients older than I8 years with no known psychiatric illnesses that could affect their responses were included.

\section{Data Analysis}

Qualitative data gathered by means of interviews were transcribed and analysed by means of the basic content analysis as described by Burnard ${ }^{25,26}$ as follows:
$*$ All the interview transcripts were read through by the researcher and general themes were identified and noted.

* Transcripts were read through again and information (relevant to the question asked) was placed under multiple headings that described the categories as they emerged (open-coding).

$*$ This list of categories was read through and similar categories were grouped together under higher-order headings in order to reduce the number of categories to a maximum of 7-12 categories. This step was repeated until the list of categories was refined to five.

* Each category was allocated a colour and the transcribed interviews were read through again and all information that fell within a certain category was marked with the corresponding colour (coding).

\section{Trustworthiness}

A colleague not familiar with the research was asked to do the same open coding and coding with the transcribed interviews as the researcher has done to ensure the researcher's interpretation of the data was accurate. To ensure that the coded information was not used out of context, regular checking and comparing was done with the original text.

Interview questions were translated using forward translation and back translation to English and Afrikaans respectively. The focus of this process was less on the linguistic/literal equivalent and more on finding a cross-cultural and conceptual match. When consensus was reached in the different language groups that it had been correctly translated, it was considered to be a valid translation as suggested by joubert et al. ${ }^{27}$.

\section{Ethical clearance and informed consent}

Ethical clearance was obtained from the Ethics Committee for Human Research at Stellenbosch University (SU) and from the Western Cape Department of Health. Consent was also obtained from the rehabilitation center's Management Committee as well as the local Community Health Center's management team.

Before giving informed consent clients were briefed on the aims of the study, the intrusion on their time as well as the information gathering procedures that would be used. Informed consent forms were signed by clients wishing to participate in the study before commencement and clients were also assured of the fact that they could, without any consequences or penalty, withdraw from the study at any time and that there would be no financial compensation for their participation.

There was minimal impact on clients' time and there were no physical or emotional risks for clients. Clients' anonymity was protected at all times by assigning a code to all participants. Where direct quotes were used these clients' codes were indicated. These codes included information pertaining to their diagnosis in order to better understand the context of the quote.

\section{RESULTS}

Clients commented extensively on the emotional impact that participating in rehabilitation had had on them as a person and on the physical changes that participation in rehabilitation had brought about in their body structure. This is reflected by the two themes that emerged from the analysis, namely experience of the service delivered and experience of the changes to self (abilities). The following flowchart (Figure I on page 62) illustrates a breakdown of the two themes and related categories and sub-categories that were gleaned from the content analysis.

\section{Experience of the service delivered}

Surprisingly, clients mainly commented on the positive feelings that arose from their rehabilitation - the changes that affected them as a person - rather than making comments that related specifically to the alleviation of body function limitations. In other words, their responses emphasised the effect of rehabilitation on their personal lives. The following three categories were identified: 


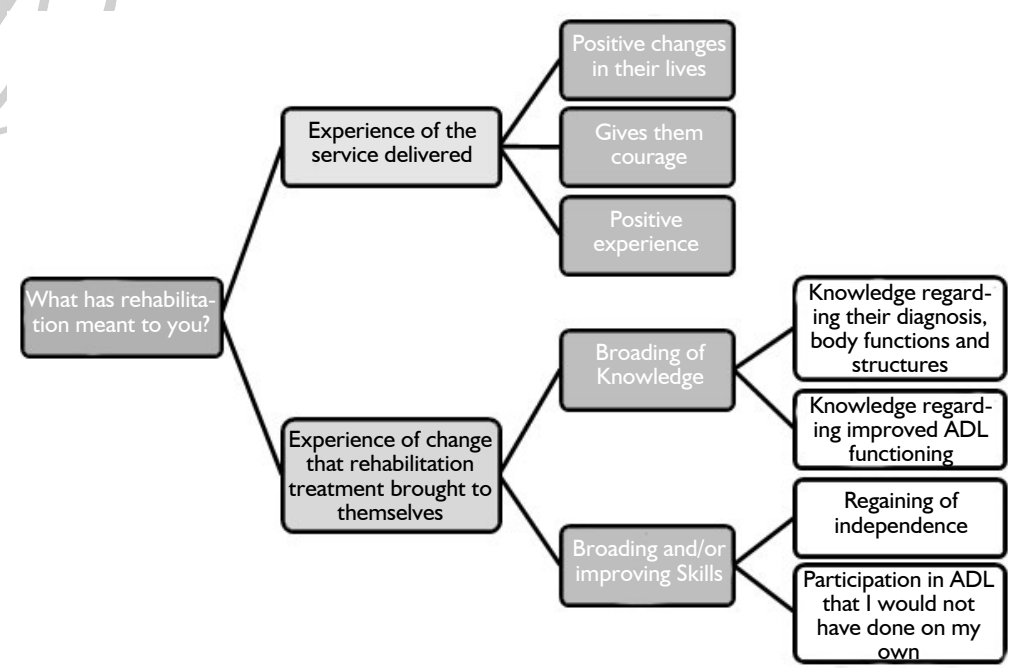

Figure I: Schematic representation of the thematic analysis of interviews

\section{Positive changes in their lives}

Comments by participants after having completed rehabilitation sessions included ...feeling like a new person, being ....more positive and ...feeling better than when they arrived at the rehabilitation centre. They seemed to appreciate the value of the rehabilitation beyond the intended physical rehabilitation outcomes and appreciated the broader implications it had had on their lives, as demonstrated in the quotes below.

If you see how I looked and how I walked when I came here, today you would say that I really am a better person and I have to thank everyone. (LBP, PI4)

For me it was like... like you could almost say I underwent a transformation...it made you feel very positive. (LBP, P4I)

If you come from the therapy you feel like a new person.(ART, P20)

\section{Gives them courage}

This category depicts the effect that rehabilitation had on clients' psychological wellbeing. They reported feeling more confident about their own abilities. Clients felt that the emotional support and encouragement provided by therapists, who also set and discussed positive outcomes with them, instilled the inner courage to strive toward attaining independence in tasks. Clients who had become despondent and had lost motivation felt that participation in rehabilitation helped them to find themselves again. It gave them hope of a better future and the courage to continue - ...one step at a time.

The therapy meant a lot to me in terms of teaching me to have more self confidence in doing things for myself. (UL, PI7)

They encouraged me and told me I should not give up hope. (CVA, P35) ...and to find myself again because I felt I was dependent on everyone. (CVA, P95)

\section{Positive experience}

The third category revealed that clients were looking forward to their therapy sessions. One might expect clients to persevere with rehabilitation merely because of the potential benefits, however, clients clearly stated that they also derived some enjoyment from therapy and looked forward to their next session. They considered rehabilitation to be important and were thankful for the level of service they received. Their experience of staff members as being helpful and friendly further contributed to their positive experience.

They are very helpful and receive us nicely. I'll put it like this: they make my day...no really they make my day and I am thankful for what I was able to learn here... (LBP, P42)

I actually looked forward to it... (CVA, P60)

\section{Experience of change that rehabilitation treatment brought to themselves}

The second theme captured the interpretation by participants regarding the goals of rehabilitation. This was evident in clients' insight in and understanding of the more tacit aims of therapy. Clients expressed what they perceived to be the benefits of therapy and the effects of rehabilitation on their ability to perform ADL. Under this theme the following two categories were identified:

\section{Broadening of knowledge}

Participants reported that they had experienced feelings of helplessness and despair on their first entry into the medical system (before referral to the rehabilitation system), as little or no information regarding their diagnosis or prognosis was provided. Participants generally reported receiving information that assisted their understanding of their condition only once they started their rehabilitation. Whilst obtaining such important knowledge was positive in terms of rehabilitation outcomes, it was of concern that clients had not understood their diagnoses better earlier on in the process.

The following quote illustrates clients helpless feeling after a stroke:

...the stroke...it really brought my life to a standstill...especially because I knew nothing about it and received no help from anywhere... and then I came to the therapy center and explained my situation to them, only then could I see the changes that started in me... (CVA, P84)

The types of knowledge internalised by participants were captured in the following two sub-categories:

Knowledge regarding their diagnosis, body functions and structures

Clients reported gaining knowledge on their diagnosis and the effect that it had on their body functions and structures. They felt that this knowledge empowered them to live with their diagnosis and to improve their well-being. Clients, especially those with chronic conditions such as Arthritis, reported that they had shared the knowledge gained in rehabilitation with family and friends. This indicated that they felt that they had gained sufficient knowledge and understanding to share this information with confidence.

They have taught me what this disease that I have is, what it means, how I should approach it, how to look after my body, how to take care of it. (ART, P25)

And that which I have learned I teach to others as well and I am glad that I could come here and could learn so that I can teach others as well. (LBP, P53)

\section{Knowledge regarding improved ADL functioning}

Knowledge regarding adaptations for improved ADL functioning as well as exercises to reduce the burden of disability was given to clients. Clients reported this to be helpful in improving independence as well as making life easier.

...showed us how... a lot of what she taught us here I can now apply and if I apply it I it makes my life much easier."(LBP, PI4)

And the exercises that she gave me to do at home, that helped me $a$ lot. (UL, P96)

\section{Broadening and/or improving skills}

Clients report improved ADL participation. This category was divided into the following two sub-categories:

\section{Regaining of independence}

Improved independence in activities as well as regained dignity was highlighted by clients as outcomes that they had achieved through 
participation in rehabilitation.

Now I can do a lot of things for myself, I don't have to depend on others. (CVA, P43)

I do not regret coming here because I can dress myself again and I can walk again - I can do things for myself, I don't have to sit and embarrass myself anymore. (CVA, P4)

The therapy meant a lot to me, because before there were a lot of things I couldn't do for myself, I was dependent on others. Now that I have received therapy, now I can do everything for myself that I couldn't do before. (UL, P92)

\section{Participation in ADL that I would not have done on my own}

For different reasons clients tended to not attempt certain ADL's after incident/diagnosis. Although clients regained varying levels of independence in ADL, their participation in rehabilitation was found to contribute to regaining that level of independence.

I learned to do things that I couldn't do. (UL, P77)

If I didn't come here that I would probably still be lying in bed and be in a wheelchair. (CVA, P48)

I thought I would never be able to ride a bicycle again and since I came here, I have been riding bicycle every night....and that is proof to me that therapy helped. And what would I have done if I didn't come to therapy? (LL, P6)

\section{DISCUSSION AND IMPLICATIONS}

The services of the rehabilitation center are aligned with the shift in the South African Healthcare system towards a primary healthcare approach. With the center's mission to provide a comprehensive inter-professional rehabilitation service close to and in clients' homes' leads to a high level of satisfaction with the service as reported by clients. Clients feel that the rehabilitation center provides appropriate input for their context and because they enjoy the therapy and look forward to their next appointment it could lead to better compliance. Also the clients' eagerness to share the knowledge they gained in therapy with others in their community could increase the community awareness of disability.

It is evident that to assess the achievement of norms and standards as set out in healthcare, that both objective and subjective information obtained from clients are indeed necessary for obtaining full understanding of the effects of rehabilitation. Subjective information is especially helpful in establishing clients' knowledge base and perception of their abilities.

Knowledge to prevent disability and empowering clients to make informed decisions regarding their healthcare is an important outcome of rehabilitation services. Subjective information made it possible to ascertain in clients' own words what they understood from the education and how they are using the knowledge in their day-to-day lives to improve their health.

Improvement in ADL functioning was clear from the results but no mention was made of the community's attitude towards clients. It is suggested that this long term goal should be explicitly investigated as it forms an essential part of community reintegration for clients.

\section{CONCLUSION}

Participation in rehabilitation was a positive and enjoyable experience for clients. Clients felt that rehabilitation inspired them to continue and also improved their confidence. This information in itself is useful to motivate the importance of providing rehabilitation services in communities. It will positively contribute towards persons with disabilities' motivation to participate in ADL.

Rehabilitation also gave clients more knowledge regarding their bodies and helped them to better understand their diagnosis and the effect it has on their bodies. They felt that rehabilitation helped them to regain their independence in ADL by providing them with the necessary knowledge and skills. If burden of disease is lessened by rehabilitation and clients are empowered to make informed decisions regarding their health it reduces the long-term impact on health and social services. Findings from this study can be used by the Western Cape Department of Health for realistic service and policy planning for persons with disability. The rehabilitation center can serve as a possible service delivery model for rehabilitation services on a primary level.

Further investigation regarding the community's attitude and environmental factors is needed to gain a full understanding of clients' reintegration into their community.

\section{ACKNOWLEDGEMENTS}

Sincere thanks to the SANPAD reference group for financial and research support.

\section{REFERENCES}

I. Department of Health. Rehabilitation for all: National Rehabilitation Policy. Pretoria: Deparment of Health. 2000.

2. Department of Health. Integrated National Disability Strategy White Paper. Pretoria: Department of Health. 1997.

3. Department of Health. Healthcare 2010: Health Western Cape's plan for ensuring equal access to quality health care. Pretoria: Department of Health. 2003.

4. Department of Health. Comprehensive Service Plan. Pretoria: Department of Health. 2007.

5. Department of Health. The Primary Health Care Package for South Africa $-\mathrm{a}$ set of norms and standards. Pretoria: Deparment of Health. 2000.

6. Department of Health. Healthcare 2020: The future of health care in the Western Cape. Draft framework. Cape Town: Department of Health. 20II.

7. Kayes NM, McPherson KM. Measuring what matters: does 'objectivity' mean good science? Disabil Rehabil, 2010; 32(I2): I0II-10I9.

8. Statistics South Africa. South African Census 2011. Pretoria. 2012.

9. Statistics South Africa. Prevalence of disability in South Africa Census 200I. Pretoria. 2005.

10. Statistics South Africa. Census 201 I: Profile of persons with disabilities in South Africa. Pretoria. 2014.

II. McColl MA, Shortt S, Godwin M, Smith K, Rowe K, O'Brien P, et al. Models for integrating rehabilitation and primary care: a scoping study. Arch Phys Med Rehabil, 2009; 90(9): 1523-1531.

12. World Health Organization. CBR: a strategy for rehabilitation, equalization of opportunities, poverty reduction and social inclusion of people with disabilities. A joint position paper. 2004.

13. Siegert RJ, Ward T, Playford ED. Human rights and rehabilitation outcomes. Disability and Rehabilitation, 2010; 32(I2): 965-97I.

14. Velema JP, Ebenso B, Fuzikawa PL. Evidence for the effectiveness of rehabilitation-in-the-community programmes. Leprosy Review, 2008; 79(I): 65-82.

15. Geddes JML, Chamberlain MA. Home-based rehabilitation for people with stroke: a comparative study of six community services providing co-ordinated, multidisciplinary treatment. Clin Rehabil, 200I; 15(6): 589.

16. Hoenig H, Lee J, Stineman M. Conceptual overview of frameworks for measuring quality in rehabilitation. Topics in Stroke Rehabilitation, 2010; 17(4): 239-25I.

17. Zhao T, Kwok JKF. Evaluating Community Based Rehabilitation: Guidelines for Accountable Practice. Rehabilitation International Regional Secretariat for Asia and the Pacific. 1999.

18. Leonardi M. Measuring health and disability: supporting policy development. The European MHADIE project. Disabil Rehabil, 20I0; 32: SI-S8.

19. McNamara C. "Basic guide to program evaluation." 1999. < http:// mcp-dev2.jbinternational.com> (I3 Oct 2006).

20. Cameron ID. Models of rehabilitation - commonalities of interventions that work and of those that do not. Disabil Rehabil, 2010; 32(I2): $105 \mathrm{I}-1058$.

21. Young D. Outcome-Based Planning and Evaluation: What are They and Why Should I Care? Rehabilitation Review, 2000; I I(5).

22. Snögren M, Sunnerhagen KS. Description of functional disability among younger stroke patients: exploration of activity and participa- 
tion and environmental factors. International Journal of Rehabilitation Research, 2009; 32(2): I 24.

23. Finkenflügel $\mathrm{H}$, Cornielje $\mathrm{H}$, Velema J. The use of classification models in the evaluation of CBR programmes. Disability \& Rehabilitation, 2008; 30(5): 348-354.

24. World Health Organization. Towards a common language for functioning, disability and health ICF. Geneva. 2002.

25. Burnard P. A method of analysing interview transcripts in qualitative research. Nurse Educ Today, I99 I; I I(6): 46 I-466.

26. Burnard P, Gill P, Stewart K, Treasure E, Chadwick B. Analysing and presenting qualitative data. Br Dent J, 2008; 204(8): 429-432.

27. Joubert G, Ehrlich R, Katzenellenbogen JM, Abdool Karim SS. Epidemiology: a research manual for South Africa. 2nd ed. Cape Town: Oxford University Press Southern Africa; 2007.

Corresponding Author

Maatje Kloppers

maatje@sun.ac.za 